\title{
On the Higher-Order Static Polarizabilities and Dispersion Coefficients of the Fullerenes: An Ab Initio Study
}

\author{
Ka Un Lao, ${ }^{1, *}$ Yan Yang, ${ }^{1, *}$ and Robert A. DiStasio Jr. ${ }^{1, \dagger}$ \\ ${ }^{1}$ Department of Chemistry and Chemical Biology, Cornell University, Ithaca, NY 14853 USA
}

(Dated: September 11, 2020)

\begin{abstract}
In this work, we used finite-field derivative techniques and density functional theory (DFT) to compute the static isotropic polarizability series $\left(\alpha_{\ell}\right.$ with $\left.\ell=1,2,3\right)$ for the $\mathrm{C}_{60}-\mathrm{C}_{84}$ fullerenes and quantitatively assess the intrinsic non-additivity in these fundamental response properties. Critical analysis of the derived effective scaling laws $\left(\alpha_{1} \sim N^{1.2}, \alpha_{2} \sim N^{2.0}, \alpha_{3} \sim N^{2.7}\right)$ provides new insight into how the electronic structure of finite-sized fullerenes - a unique dichotomy of electron confinement and delocalization effects due to their quasi-spherical cage-like structures and encapsulated void spaces - simultaneously limits and enhances their quantum mechanical response to electric field perturbations. Corresponding molecular dispersion coefficients $\left(C_{n}\right.$ with $\left.n=6,8,9,10\right)$ needed to describe the non-trivial van der Waals (vdW) interactions in fullerene-based systems were obtained by inputting the $\alpha_{\ell}$ into the hollow sphere model within the modified single-frequency approximation. Using first-order perturbation theory in conjunction with $>140,000$ DFT calculations, we also computed the non-negligible zero-point vibrational contributions to $\alpha_{1}$ in $\mathrm{C}_{60}$ and $\mathrm{C}_{70}$, thereby enabling direct comparison between theory and experiment for these quintessential nanostructures.
\end{abstract}

The molecular polarizability $\left(\alpha_{\ell}\right)$ describes the tendency of a molecule to form an induced multipole moment in the presence of an electric field $\left(\alpha_{1}\right.$, dipole polarizability), field gradient $\left(\alpha_{2}\right.$, quadrupole polarizability), field Laplacian $\left(\alpha_{3}\right.$, octupole polarizability), and/or higher field derivatives [1-4]. Knowledge of the $\alpha_{\ell}$ series is crucial when describing induction and dispersion/vdW interactions [4-6], predicting/understanding the spectroscopic signatures (i.e., Raman, sum-frequency generation) of molecules and condensed matter [7, 8], as well as developing next-generation polarizable force fields and machine-learning based intra-/inter-molecular potentials. As quantum mechanical response properties, the $\alpha_{\ell}$ series is governed by complex many-body interactions (e.g., electron correlation, charge delocalization, secondary polarization), and tends to become more non-additive with increasing order and molecular size/complexity [1-4, 9-13]. From a theoretical point of view, an accurate and reliable description of $\alpha_{\ell}$ can be quite demanding, and often requires sophisticated treatment of electron correlation in conjunction with large (and diffuse) basis sets [14-19]. From an experimental point of view, $\alpha_{\ell}$ measurements are susceptible to (zeropoint) vibrational contributions, thermal effects, as well as origin and orientational dependencies [20-22].

Through the Casimir-Polder (CP) relationship [23], non-additivity in $\alpha_{\ell}$ is also reflected in the dispersion coefficients $\left(C_{n}\right)$, which govern the strength of the $\mathrm{vdW}$ forces between molecules and materials. Since even slight variations in the (effective) vdW power laws can impact the structure, stability, and properties of a system [24$26]$, knowledge of how $\alpha_{\ell}$ scales with system size is funda-

\footnotetext{
* These authors contributed equally to this work.

$\dagger$ distasio@cornell.edu
}

mental to understanding these ubiquitous non-bonded interactions. At the nanoscale, non-additivity in $C_{n}$ is particularly important, as vdW forces are largely responsible for directing self-assembly and the energetic contributions from higher-order terms can be $50 \%$ of the leadingorder $C_{6}$ component [27-29]. Given that fullerenes, nanotubes, and multi-layer graphene already exhibit unusual scaling behavior [24-26, 30-36] at the $C_{6}$ level, enhanced non-additivity in the higher-order $C_{n}$ is expected to have an even more profound effect on such nanostructures.

Of specific interest here are the fullerenes, which are characterized by quasi-spherical cage-like structures, encapsulated void spaces, and nearly uniform surface electron densities, thereby making them unique systems for studying non-additivity in the $\alpha_{\ell}$ and $C_{n}$ scaling landscapes $[24,37-40]$. For the popular fullerenes (e.g., $\mathrm{C}_{60}$ and $\left.\mathrm{C}_{70}\right), \alpha_{1}$ and $C_{6}$ have been well-investigated by theory $[24,38,41-43]$ and experiment $[22,44-49]$, with most studies reporting qualitatively similar values for these leading-order terms (although direct comparison between theory and experiment has not been possible due to vibrational and thermal effects [20-22]). Theoretical studies $[24,38,40]$ have also shown that $\alpha_{1}$ and $C_{6}$ exhibit strong non-additivity with increasing fullerene size as well as non-trivial quantum-/finite-size effects $[39,50]$. However, there has been little to no work dedicated to the higher-order $\alpha_{\ell}$ and $C_{n}$ for the fullerenes, and many existing models [37, 51-57] for these quantities - the veracity of which have yet to be confirmed - still rely on accurate values for $\alpha_{1}$ (or even $\alpha_{\ell}$ ) as input.

In this Letter, we address these limitations by computing ab initio values for the $\alpha_{\ell}$ series $(\ell=1,2,3)$ in the $\mathrm{C}_{60}-\mathrm{C}_{84}$ fullerenes using DFT and finite-field derivative techniques. With these values in hand-which are arguably the most accurate values to date - we obtain the molecular dispersion coefficients $\left(C_{n}\right.$ with $\left.n=6,8,9,10\right)$, 
quantitatively ascertain the intrinsic non-additivity in the $\alpha_{\ell}$ (and $C_{n}$ ) series, and derive the corresponding effective scaling laws as a function of fullerene size. Detailed analysis of the $a b$ initio data with respect to classical models that treat the fullerenes as conducting spherical shells or solid spheres demonstrates how the unique electronic structure of single-walled fullerenes - a complex dichotomy between electron confinement and charge delocalization - simultaneously limits and enhances their response to electric field perturbations. Analogous to the deviations from asymptotic vdW scaling laws observed at finite (nanoscale) distances, this analysis also demonstrates how quantum-/finite-size effects markedly alter the $\alpha_{\ell}$ (and $C_{n}$ ) scaling landscapes in these quintessential nanostructures. We conclude this work by computing the non-negligible $(\approx 1-2 \%)$ zero-point vibrational corrections $[20,58-60]$ to $\alpha_{1}$ for the $\mathrm{C}_{60}$ and $\mathrm{C}_{70}$ fullerenes, enabling the first direct comparison between experiment and theory for this fundamental response property.

To begin, we computed static isotropic (electronic) polarizabilities ( $\alpha_{\ell}$ with $\ell=1,2,3$ ) for the lowestenergy isomers of the $\mathrm{C}_{60}, \mathrm{C}_{70}, \mathrm{C}_{76}, \mathrm{C}_{78}$, and $\mathrm{C}_{84}$ fullerenes using finite-field derivatives at the SCAN0/Sadlej//SCAN0/6-31G(d) level (see Supporting Information (SI) for computational details, Table S1 for isomer comparison, and Tables S7-S11 for the optimized structures). Since the SCAN0 [61] hybrid functional, which admixes $25 \%$ exact exchange into the SCAN [62] meta-GGA functional, combined with the Sadlej [14] triple- $\zeta$ basis set, which has been optimized for molecular properties such as moments/polarizabilities, furnishes $\alpha_{\ell}$ values to $\approx 1 \%$ of benchmark quantum chemical methods for $\mathrm{C}_{20}$ (the smallest cage-like fullerene [63]), this level of theory was used for the larger fullerenes in this work (Table S2). Corresponding frequency-dependent polarizabilities $\left(\alpha_{\ell}(i u)\right)$ were obtained within the modified single-frequency approximation (MSFA) [56] by inputting $\alpha_{\ell}$ into the hollow sphere (HS) model of Tao and Perdew [54], and used to compute $C_{n}(n=6,8,9,10)$ via the CP integral [23]. All calculations were performed using Q-Chem [64] and FHI-aims [65].

The computed $\alpha_{\ell}$ (and $C_{n}$ ) are summarized in Table I (and Table S3). For $\alpha_{1}$ and $C_{6}$, our values are in excellent agreement with the available theoretical data for the fullerenes (Table S6). For the higher-order terms, $\alpha_{2}$ and $\alpha_{3}$ in Table I are the only ab initio values available to date. In previous studies [37, 40, 53-57], these quantities have been estimated using a classical formula derived by considering a conducting spherical shell (or solid sphere) of uniform electron density with outer radius $R$ and thickness $t$, namely, $\alpha_{\ell} \approx \alpha_{\ell}^{\text {model }}=R^{2 \ell+1}$ (valid for $0<t \leq R)[37,66]$. Since these are among the simplest models for the fullerenes [37, 50], we first examine the models' assumption that $\alpha_{\ell}$ can be derived using a single radius per fullerene by inverting this formula to obtain $R_{\ell}=\alpha_{\ell}^{1 /(2 \ell+1)}$. These $R_{\ell}$ values - along with the esti-
TABLE I. Values (in au) for the multipole polarizabilities $\left(\alpha_{\ell}\right.$ with $\ell=1,2,3$; computed at the SCAN0/Sadlej//SCAN0/6$31 \mathrm{G}(\mathrm{d})$ level $)$ and homo-molecular dispersion coefficients $\left(C_{n}\right.$ with $n=6,8,10$; computed by inputting $\alpha_{\ell}$ in the HS/MSFA model) in the $\mathrm{C}_{60}-\mathrm{C}_{84}$ fullerenes. Also provided are the effective scaling law parameters (and $R^{2}$ values) from non-linear fits to a power law $\left(a N^{b}\right)$ ansatz. Additional homo-/heteromolecular $C_{n}(n=6,8,9,10)$ can be found in Tables S4-S5.

\begin{tabular}{c|rrr|rrr}
\hline \hline & \multicolumn{3}{|c|}{ Ab Initio } & \multicolumn{3}{c}{ Ab Initio + HS/MSFA } \\
\hline Molecule & $\alpha_{1} / 10^{2}$ & $\alpha_{2} / 10^{4}$ & $\alpha_{3} / 10^{6}$ & $C_{6} / 10^{5}$ & $C_{8} / 10^{7}$ & $C_{10} / 10^{9}$ \\
\hline $\mathrm{C}_{60}\left(I_{\mathrm{h}}\right)$ & 5.368 & 4.281 & 3.319 & 0.990 & 4.207 & 14.620 \\
$\mathrm{C}_{70}\left(D_{5 \mathrm{~h}}\right)$ & 6.640 & 5.803 & 5.153 & 1.462 & 6.891 & 26.963 \\
$\mathrm{C}_{76}\left(D_{2}\right)$ & 7.250 & 6.877 & 6.543 & 1.735 & 8.861 & 37.418 \\
$\mathrm{C}_{78}\left(C_{2 \mathrm{v}}\right)$ & 7.546 & 7.224 & 6.915 & 1.863 & 9.639 & 41.139 \\
$\mathrm{C}_{84}\left(D_{2 \mathrm{~d}}\right)$ & 8.090 & 8.310 & 8.345 & 2.143 & 11.872 & 53.827 \\
\hline$a$ & 3.903 & 13.487 & 54.633 & 10.231 & 165.632 & 2461.939 \\
$b$ & 1.206 & 1.970 & 2.696 & 2.248 & 3.045 & 3.816 \\
$R^{2}$ & 0.994 & 1.000 & 0.998 & 0.996 & 0.999 & 0.999 \\
\hline \hline
\end{tabular}

mated (outer) physical radius of each fullerene $\langle R\rangle[67]$ are plotted against $N$ in Fig. 1. From this figure, one can see that $R_{1} \approx R_{2} \approx R_{3} \approx\langle R\rangle$ for $\mathrm{C}_{60}-\mathrm{C}_{84}$; with mean signed deviations of $-2.9 \%\left(R_{1}\right),+0.2 \%\left(R_{2}\right)$, and $+1.5 \%$ $\left(R_{3}\right)$, each $R_{\ell}$ agrees fairly well with $\langle R\rangle$, and the emerging picture is qualitatively consistent with these models. In the same data, we also observe a weak (but still increasing) dependence of $R_{\ell}$ on $\ell$ as $R_{3} \gtrsim R_{2} \gtrsim R_{1}$ for each fullerene. Here, we argue that both of these observations can be rationalized by considering the unique electronic structure of the fullerenes, whose quasi-spherical cage-like structures and encapsulated void spaces lead to an essentially metallic electron density that is delocalized across the entire fullerene surface, yet largely confined to a thin surrounding shell. Unlike the spherical shell and solid sphere models - in which the density is confined to an infinitesimally thin shell at the conductor surface [37] - electrons on the fullerene surface still retain some degree of radial flexibility, which serves to enhance their response to electric field perturbations. This property manifests itself in the (albeit weak) growth of $R_{\ell}$ with $\ell$ observed above, which indicates enhanced nonadditivity in the fullerenes beyond that due to the increasingly higher-order powers in the model $\alpha_{\ell}$ formula. In the same breath, the fact that the electrons on the fullerene surface are largely confined to a surrounding thin shell also simultaneously limits the radial extent of their response to external electric fields. Evidence of this is seen in the qualitatively similar $R_{\ell}$ values in Fig. 1 that only weakly depend on $\ell$; although higher-order $\alpha_{\ell}$ data is scarce, the dependence of $R_{\ell}$ on $\ell$ seems to be stronger for extended systems like $n$-alkanes [68], and is expected to be even more pronounced in conjugated extended systems like s-trans alkenes and polyacenes [69]. 

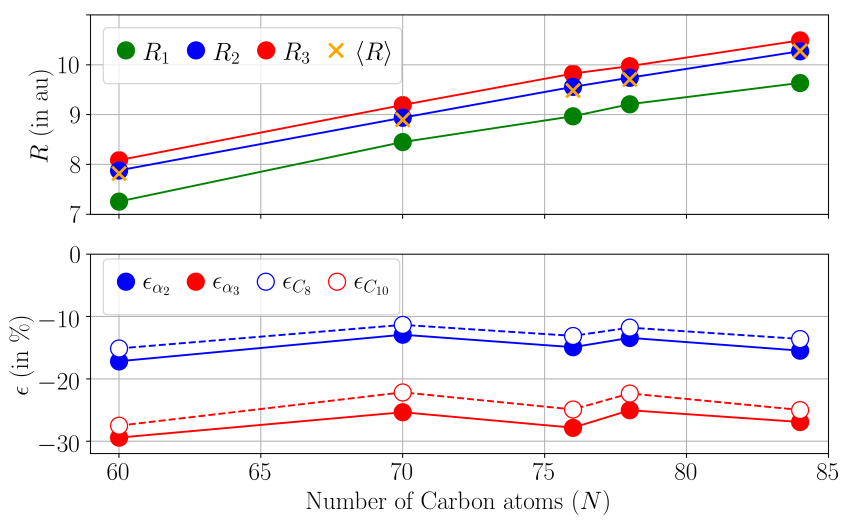

FIG. 1. Top: System-size dependence of the $\ell$-dependent radii (computed using the $a b$ initio $\alpha_{\ell}$ in the conducting spherical shell/solid sphere model formula, $\left.R_{\ell}=\alpha_{\ell}^{1 /(2 \ell+1)}\right)$ and estimated physical radii $(\langle R\rangle)[67]$ in the $\mathrm{C}_{60}-\mathrm{C}_{84}$ fullerenes. Bottom: Percent error when predicting higher-order $\alpha_{\ell}(\ell=2,3)$ and $C_{n}(n=8,10)$ under the model assumption of a single radius $\left(R=R_{1}\right)$ per fullerene.

Although the differences among $R_{\ell}$ are small, the errors made when predicting $\alpha_{\ell}$ (and $C_{n}$ ) under the assumption of a single radius per fullerene can be substantial and warrant further discussion. Since an accurate determination of $\alpha_{1}$ is most straightforward among the $\alpha_{\ell}$ series, we first set $R=R_{1}=\alpha_{1}^{1 / 3}$ for each fullerene (following previous work in Refs. $[40,53,55-57,66]$ ) and re-compute $\alpha_{2}$ and $\alpha_{3}$ via $\alpha_{\ell} \approx \alpha_{\ell}^{\text {model }}=R^{2 \ell+1}$. The corresponding errors, $\epsilon_{\alpha_{\ell}} \equiv\left(\alpha_{\ell}^{\text {model }}-\alpha_{\ell}\right) / \alpha_{\ell}$, are also plotted in Fig. 1, from which one can immediately see that these higher-order properties are substantially underestimated in $\mathrm{C}_{60}-\mathrm{C}_{84}$. With mean deviations of $14.8 \pm 1.5 \%$ $\left(\alpha_{2}\right)$ and $26.9 \pm 1.6 \%\left(\alpha_{3}\right)$, these errors are significant in magnitude and increase with $\ell$; such discrepancies can be traced back to the variability in $R_{\ell}\left(e . g ., R_{3} \gtrsim R_{2} \gtrsim R_{1}\right)$, which becomes amplified by the increasingly higher-order powers in the model formula. Also depicted are the errors which propagate into $C_{8}$ and $C_{10}$ when inputting these $\alpha_{\ell}$ values into the HS/MSFA model; with mean deviations of $13.0 \pm 1.4 \%\left(C_{8}\right)$ and $24.4 \pm 2.0 \%\left(C_{10}\right)$, the non-additivity in these quantities is also substantially underestimated, rendering them unsuitable for describing $\mathrm{vdW}$ interactions between fullerenes. Here, the errors in $C_{8}$ and $C_{10}$ are quite similar to those in $\alpha_{2}$ and $\alpha_{3}$, which follows from factoring $\alpha_{\ell}$ out of the CP integral (which yields $C_{8} \propto \alpha_{1} \alpha_{2}$ and $\left.C_{10} \propto \alpha_{1} \alpha_{3}+\alpha_{2} \alpha_{2}\right)[40,54]$. We further note that the $R$ used to generate the error profile in Fig. 1 is not unique. Setting $R=\langle R\rangle$ is also physically justifiable, and overestimates $\alpha_{1}$ by $9.4 \%$ and underestimates $\alpha_{2}$ and $\alpha_{3}$ by $1.1 \%$ and $10.0 \%$, respectively [70]; since $\langle R\rangle \approx R_{2}$, the error is still substantial and simply redistributed onto $\alpha_{1}$ and $\alpha_{3}$. Another logical choice for $R$ arises from treating each fullerene as a spherical dielectric shell [71] with $\varepsilon$ derived from the HOMO-LUMO gap [50, 72]; for $\mathrm{C}_{60}$, this yields an $R$ that is only $1.5 \%$ larger than $R_{1}$ and hence a similar error profile to that in Fig. 1. Alternatives based on the current theory of $\mathrm{vdW}$ radii might also be interesting to consider [73, 74].

As the system size increases, the error introduced by using a single radius to predict $\alpha_{2}$ and $\alpha_{3}$ (or $C_{8}$ and $C_{10}$ ) is fairly constant and does not increase with $N$ (see Fig. 1). Such system-size-independent deviations are reflected in the relatively small $(\approx 1.5 \%)$ standard deviations in $\epsilon_{\alpha_{\ell}}$ (and $\epsilon_{C_{n}}$ ), and are primarily due to the fact that the variability among $R_{\ell}$ values does not increase with $N$ for $\mathrm{C}_{60}-\mathrm{C}_{84}\left(c f . R_{2} / R_{1}=1.04\right.$ (1.03) and $R_{3} / R_{1}=1.05$ (1.05) for $\left.\mathrm{C}_{60}\left(\mathrm{C}_{84}\right)\right)$. Physically speaking, this can again be attributed to the fullerene electronic structure, in which the electron density is largely confined to a thin shell whose thickness does not grow with $N[40,50,56]$. In the same breath, this sizeindependence is also due in part to treating the fullerene as a molecule instead of a collection of atoms [56]; in doing so, many-body interactions and electron delocalization effects - both of which are size-dependent by definition and largely responsible for the non-additivity in $\alpha_{\ell}$-are intrinsically accounted for by the model. In any case, the use of a single radius (per fullerene) is simply not flexible enough for a quantitative prediction of $\alpha_{\ell}$ and $C_{n}$; when computing $C_{n}$ (in particular), more sophisticated models that account for the non-trivial $\ell$ dependence of $R_{\ell}$ (e.g., HS/MSFA [54, 56]) - in conjunction with the high-quality ab initio $\alpha_{\ell}$ values provided herein - are crucial for an accurate and reliable description of these quintessential nanostructures.

To further explore how $\alpha_{\ell}\left(C_{n}\right)$ scale with fullerene size, we now derive the corresponding effective scaling laws by fitting our data to power law $\left(a N^{b}\right)$ ansätze. The resulting parameters ( $a$ and $b$ ) and $R^{2}$ values are provided in Table I (with fitted functions plotted in Fig. S1). With $R^{2}>0.99$ in all cases, these fits accurately describe the system-size dependence in these quantities, yielding negligible fitting errors of $-0.05 \%,-0.02 \%,-0.22 \%$ for $\alpha_{1}, \alpha_{2}, \alpha_{3}\left(-0.21 \%,-0.22 \%,-0.38 \%\right.$ for $\left.C_{6}, C_{8}, C_{10}\right)$. Here, we find that $\alpha_{1}, \alpha_{2}$, and $\alpha_{3}$ scale as $\sim N^{1.2}, \sim N^{2.0}$, and $\sim N^{2.7}$ for the $\mathrm{C}_{60}-\mathrm{C}_{84}$ fullerenes; with exponents that substantially deviate from unity, these terms are all strongly non-additive and become increasingly more so for larger $\ell$. For $\alpha_{1}$, our finding that $b=1.21$ is in excellent agreement with Kauczor et al. $(b=1.25)$ [38] and Tao et al. $(b=1.19)$ [40] (see Table II); due to the scarcity of higher-order $\alpha_{\ell}$, the $b$ values obtained for $\alpha_{2}$ and $\alpha_{3}$ herein are the only ab initio values available to date. We note in passing that further comparisons to the values provided by Saidi et al. [39] and Tao et al. [40] would not be appropriate, as these values correspond to a much wider range of finite-sized fullerenes (e.g., $\mathrm{C}_{60}-\mathrm{C}_{720}$ and $\mathrm{C}_{60}-\mathrm{C}_{3840}$ ); to characterize how quantum-/finite-size effects influence the $\alpha_{\ell}$ (and $C_{n}$ ) scaling landscape for $\mathrm{C}_{60}-\mathrm{C}_{84}$, we now discuss our findings with respect to the 
TABLE II. Effective scaling law exponents $(b)$ for the polarizabilities $\left(\alpha_{\ell}\right)$ and dispersion coefficients $\left(C_{n}\right)$ in the fullerenes.

\begin{tabular}{|c|c|c|c|c|c|c|}
\hline & $\alpha_{1}$ & $\alpha_{2}$ & $\alpha_{3}$ & $C_{6}$ & $C_{8}$ & $C_{10}$ \\
\hline$A b$ Initio $^{a}$ & $1.21^{b}$ & $1.97^{b}$ & $2.70^{b}$ & - & - & - \\
\hline Ab Initio + HS/MSFA ${ }^{a}$ & - & - & - & $2.25^{b}$ & $3.05^{b}$ & $3.82^{b}$ \\
\hline 2 (spherical shel & $1.50^{b}$ & $2.50^{b}$ & $3.50^{b}$ & $2.75^{d}$ & $3.75^{d}$ & $4.75^{d}$ \\
\hline$N \propto R^{3}$ (solid sp & $1.00^{b}$ & $1.67^{b}$ & $2.33^{b}$ & $2.00^{d}$ & $2.67^{d}$ & $3.33^{d}$ \\
\hline & - & - & - & 2.25 & - & - \\
\hline al. $[38]^{g}$ & 1.25 & - & - & 2.19 & - & - \\
\hline 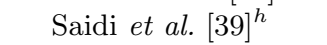 & 1.46 & - & - & 2.80 & - & - \\
\hline Tao et al. $[40]^{i}$ & 1.19 & 1.65 & 2.11 & 2.26 & 2.73 & 3.20 \\
\hline
\end{tabular}

${ }^{a} \mathrm{C}_{60}-\mathrm{C}_{84}$ fullerenes ${ }^{b}$ This work ${ }^{c}$ Conducting spherical shell model; $R \rightarrow \infty$ limit ${ }^{d}$ Ref. [37] ${ }^{e}$ Conducting solid sphere model; all $R$ values ${ }^{f}$ Self-consistent screening (SCS) model; $\mathrm{C}_{20}-\mathrm{C}_{540}{ }^{g}$ Time-dependent DFT (B3LYP); $\mathrm{C}_{60}-\mathrm{C}_{84}{ }^{h}$ Capacitance-polarizability interaction (CPI) model; $\mathrm{C}_{60}-\mathrm{C}_{720}{ }^{i}$ Conducting spherical shell/solid sphere model (for $\alpha_{\ell}$ ) and HS/SFA (for $C_{n}$ ); $\mathrm{C}_{60}-\mathrm{C}_{3840}$ (two-point formula)

formal $(R \rightarrow \infty)$ limits of the conducting spherical shell and solid sphere models.

Since $N \propto R^{2}$ for a spherical shell (in the $R \rightarrow \infty$ limit) and $N \propto R^{3}$ for a solid sphere (for all $R$ values), these models differ in their description of how $\alpha_{\ell}$ grows with $N$, i.e., $\alpha_{\ell}=R^{2 \ell+1}=N^{(2 \ell+1) / \delta}$ with $\delta=2$ (spherical shell) and $\delta=3$ (solid sphere). The corresponding scaling law exponents for $\alpha_{\ell}$ (and $C_{n}$ ) based on these models are provided in Table II, from which one can see that the growth of $\alpha_{\ell}$ with $N$ in the solid sphere model is slower than that in the $\mathrm{C}_{60}-\mathrm{C}_{84}$ fullerenes, which in turn is markedly slower than that predicted by the $R \rightarrow \infty$ limit of the spherical shell model. Again, both of these observations can be rationalized by considering the dichotomous electronic structure of the fullerenes, which simultaneously limits and enhances their response to electric field perturbations. On one hand, confinement of the electrons to a thin shell unusually far from the fullerene center leads to a substantially more polarizable electron density than the distribution of the same number of electrons throughout the volume of a solid sphere (with a necessarily smaller $R$ ), thereby leading to enhanced $\alpha_{\ell}$ values compared to the solid sphere model. On the other hand, the curvature in the finite-sized fullerenes suppresses many-body polarization of the electrons across the surface, which limits the extent of the electronic response and leads to reduced $\alpha_{\ell}$ values compared to the graphene-like $R \rightarrow \infty$ limit of the spherical shell model.

For the $C_{n}$, we find that $C_{6}, C_{8}$, and $C_{10}$ scale as $\sim N^{2.2}, \sim N^{3.0}$, and $\sim N^{3.8}$, which is again indicative of strong non-additivity that increases with $\ell$. Since this non-additivity primarily originates from the intrinsic non-additivity in the underlying $\alpha_{\ell}$, our discussion of the $C_{n}$ scaling laws will be brief. For $C_{6}$, our finding that $b=2.25$ is in excellent agreement with previous studies employing time-dependent DFT $(b=2.19)[38]$ as well as the SCS $(b=2.25)[24]$ and HS/SFA $(b=2.26)[40]$ models (Table II). Following the analysis performed above for $\alpha_{\ell}$, we again find that our results lie between the $N^{n / 3}$ and $N^{(n-1 / 2) / 2}$ dependence of the solid sphere and spherical shell models [37]; as such, our data unambiguously confirms that the $C_{n}$ in $\mathrm{C}_{60}-\mathrm{C}_{84}$ grow much faster than a pairwise-additive model would suggest.

Returning to the $a b$ initio $\alpha_{\ell}$ provided herein, we conclude this Letter by investigating the zero-point vibrational contributions (zpvc) to $\alpha_{1}$ in the popular $\mathrm{C}_{60}$ and $\mathrm{C}_{70}$ fullerenes, as these often non-negligible contributions enable direct comparison between experiment and theory. For a molecule in its ground vibrational state, $\alpha_{1}^{\mathrm{zpvc}}$ can be computed using first-order perturbation theory [58-60] as $\alpha_{1}^{\text {zpvc }}=\sum_{i} \kappa_{i}$, in which $\kappa_{i} \equiv \frac{1}{4}\left[\left(\frac{\partial^{2} \alpha_{1}}{\partial q_{i}^{2}}\right)_{0}-\sum_{j} \frac{\phi_{i i j}}{\omega_{j}}\left(\frac{\partial \alpha_{1}}{\partial q_{j}}\right)_{0}\right]$ is the contribution from the $i$-th vibrational mode. In this expression, $\left(\partial^{n} \alpha_{1} / \partial q_{j}^{n}\right)_{0}$ are the $n$-th partial derivatives of $\alpha_{1}$ (evaluated at the equilibrium structure) with respect to normal mode $q_{j}$ with frequency $\omega_{j}, \phi_{i i j}$ are the anharmonic (cubic) force constants, and the sum includes all vibrational modes; see SI and Ref. [18] for more details. To obtain $a b$ initio values for these quantities, we performed an extensive series of $(>140,000)$ DFT calculations, and found that $\alpha_{1}^{\mathrm{zpvc}}=8.5 \mathrm{au}\left(\mathrm{C}_{60}\right)$ and $9.6 \mathrm{au}$ $\left(\mathrm{C}_{70}\right)$; these vibrational contributions are non-negligible in magnitude and account for $\approx 1.6 \%$ and $\approx 1.4 \%$ of $\alpha_{1}$. Individual contributions $\left(\kappa_{i}\right)$ to $\alpha_{1}^{\text {zpvc }}$ from each vibrational mode in $\mathrm{C}_{60}$ are plotted in Fig. 2. Similar to $\alpha_{1}^{\text {zpvc }}$ in water [18], the primary vibrational contributions are due to high-frequency bond-stretching modes [75] (i.e., $\approx 1400-1600 \mathrm{~cm}^{-1}$; largest contribution from the $\mathrm{H}_{\mathrm{u}}$ mode at $\left.\omega=1564 \mathrm{~cm}^{-1}\right)$, and not low-frequency squashing $\left(\approx 250 \mathrm{~cm}^{-1}\right)$ and/or breathing $\left(\approx 500 \mathrm{~cm}^{-1}\right)$ modes [76-78]. From Table III, one can see that $\alpha_{1}$ is slightly smaller than the DOSD estimate $[22,48]$ for $\mathrm{C}_{60}$, while $\alpha_{1}^{\text {tot }}=\alpha_{1}+\alpha_{1}^{\text {zpvc }}$ are well within the error bars measured by molecular beam deflection $[44,46]$ and time-

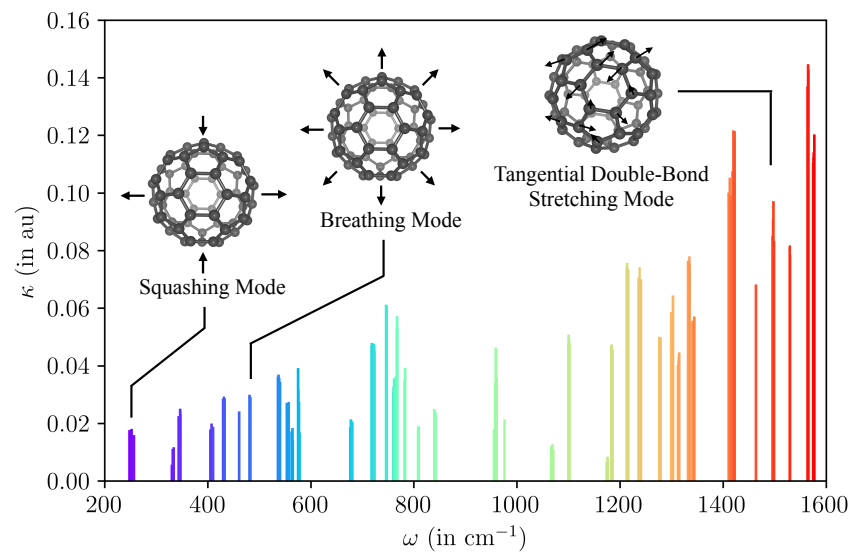

FIG. 2. Individual mode contributions ( $\kappa$ in au) to $\alpha_{1}^{\text {zpvc }}$ in $\mathrm{C}_{60}$ (computed at the SCAN/tier-1//SCAN/tier-1 level). The corresponding plot for $\mathrm{C}_{70}$ is provided in Fig. $\mathrm{S} 2$. 
TABLE III. Summary of the ab initio electronic $\left(\alpha_{1}\right.$, computed at the SCAN0/Sadlej//SCAN0/6-31G(d) level), vibrational $\left(\alpha_{1}^{\text {zpvc }}\right.$, computed at the SCAN/tier-1//SCAN/tier-1 level $)$, and total $\left(\alpha_{1}^{\text {tot }} \equiv \alpha_{1}+\alpha_{1}^{\text {zpvc }}\right)$ polarizabilities for $\mathrm{C}_{60}$ and $\mathrm{C}_{70}$ along with the available experimental data.

\begin{tabular}{c|ccc|c}
\hline \hline & \multicolumn{3}{|c|}{ Theory } & Experiment \\
\hline & $\alpha_{1}$ & $\alpha_{1}^{\text {zpvc }}$ & $\alpha_{1}^{\text {tot }}$ & $\alpha_{1}^{\text {tot }}$ \\
\hline $\mathrm{C}_{60}$ & 536.8 & 8.5 & 545.3 & $516.2 \pm 54^{a}, 533.1 \pm 27^{b}, 599.9 \pm 41^{c}$ \\
& & & & $558.6 \pm 17^{d}, 589.8 \pm 20^{e}$ \\
\hline $\mathrm{C}_{70}$ & 663.8 & 9.6 & 673.4 & $688.3 \pm 95^{a}, 732.2 \pm 55^{c}, 718.0 \pm 9^{e}$ \\
\hline
\end{tabular}

$\overline{{ }^{a} \text { Molecular beam deflection }[44,46]^{b} \text { Time-of-flight spectrometry [45] }}$

${ }^{c}$ Matter-wave interferometry (MWI) [47] ${ }^{d}$ Dipole oscillator strength distribution (DOSD) estimate of $\alpha_{1}[22,48]{ }^{e}$ MWI [49]

of-flight spectrometry [45] for $\mathrm{C}_{60}$ and $\mathrm{C}_{70}$. While our values are consistently smaller than those obtained using matter-wave interferometry [47, 49], the experimental ratios of $\alpha_{1}^{\text {tot, } \mathrm{C}_{70}} / \alpha_{1}^{\text {tot, } \mathrm{C}_{60}}=1.22$ (believed to be more accurate than absolute $\alpha_{1}$ ) using this technique are in excellent agreement with our ab initio value of 1.23.

The $\alpha_{\ell}$ (and $C_{n}$ ) provided herein for $\mathrm{C}_{60}-\mathrm{C}_{84}$ are the most accurate and reliable theoretical values obtained to date, and unequivocally demonstrate that these quantities are strongly non-additive and become increasingly more so for larger $\ell$ (and $n$ ). Derivation of the corresponding effective scaling laws in addition to a critical analysis of the $\alpha_{\ell}$ and $C_{n}$ data in the context of the classical spherical shell and solid sphere models provides new insight into how the unique electronic structure of the single-walled fullerenes - a complex interplay between electron confinement effects and charge delocalization due to the structure and topology of these nanosystems - serves to both limit and enhance their response to electric field perturbations. Of particular interest are the quantum-/finite-size effects observed in these scaling laws, which are analogous (and intimately related) to the deviations from asymptotic vdW scaling laws observed in both finite and extended systems at nanoscale distances [25, 26, 30-36, 79], and undoubtedly impact the structure, function, and properties of these quintessential nanostructures.

All authors acknowledge helpful scientific discussions with Hsin-Yu Ko, Alexandre Tkatchenko, and Yang Yang. This work was primarily supported by the Cornell Center for Materials Research with funding from the NSF MRSEC program (DMR-1719875). This research used resources of the National Energy Research Scientific Computing Center, which is supported by the Office of Science of the U.S. Department of Energy under Contract No. DE-AC02-05CH11231. This research used resources of the Argonne Leadership Computing Facility at Argonne National Laboratory, which is supported by the Office of Science of the U.S. Department of Energy under Contract No. DE-AC02-06CH11357.
[1] K. D. Bonin and V. V. Kresin, Electric-Dipole Polarizabilities of Atoms, Molecules, and Clusters (World Scientific, Singapore, 1997).

[2] G. Maroulis, Atoms, Molecules and Clusters in Electric Fields: Theoretical Approaches to the Calculation of Electric Polarizability (World Scientific, Singapore, 2006).

[3] G. Maroulis, Computational Aspects of Electric Polarizability Calculations: Atoms, Molecules and Clusters (IOS Press, Amsterdam, 2006).

[4] A. J. Stone, The Theory of Intermolecular Forces, 2nd ed. (Oxford University Press, Oxford, 2013).

[5] S. Grimme, A. Hansen, J. G. Brandenburg, and C. Bannwarth, "Dispersion-corrected mean-field electronic structure methods," Chem. Rev. 116, 5105-5154 (2016).

[6] J. Hermann, R. A. DiStasio Jr., and A. Tkatchenko, "First-principles models for van der Waals interactions in molecules and materials: Concepts, theory, and applications," Chem. Rev. 117, 4714-4758 (2017).

[7] S. Luber, M. Iannuzzi, and J. Hutter, "Raman spectra from ab initio molecular dynamics and its application to liquid s-methyloxirane," J. Chem. Phys. 141, 094503 (2014).

[8] G. R. Medders and F. Paesani, "Dissecting the molecular structure of the air/water interface from quantum simulations of the sum-frequency generation spectrum," J. Am. Chem. Soc. 138, 3912-3919 (2016).

[9] H.-Y. Kim, J. O. Sofo, D. Velegol, M. W. Cole, and G. Mukhopadhyay, "Static polarizabilities of dielectric nanoclusters," Phys. Rev. A 72, 053201 (2005).

[10] H.-Y. Kim, J. O. Sofo, D. Velegol, M. W. Cole, and A. A. Lucas, "van der Waals forces between nanoclusters: Importance of many-body effects," J. Chem. Phys. 124, 074504 (2006).

[11] A. Mayer, "Formulation in terms of normalized propagators of a charge-dipole model enabling the calculation of the polarization properties of fullerenes and carbon nanotubes," Phys. Rev. B 75, 045407 (2007).

[12] H.-Y. Kim, J. O. Sofo, D. Velegol, M. W. Cole, and A. A. Lucas, "van der Waals dispersion forces between dielectric nanoclusters," Langmuir 23, 1735-1740 (2007).

[13] T. Helgaker, S. Coriani, P. Jørgensen, K. Kristensen, J. Olsen, and K. Ruud, "Recent advances in wave function-based methods of molecular-property calculations," Chem. Rev. 112, 543-631 (2012).

[14] A. J. Sadlej, "Medium-size polarized basis sets for highlevel correlated calculations of molecular electric properties," Collect. Czech. Chem. Commun. 53, 1995-2016 (1988).

[15] D. E. Woon and T. H. Dunning Jr., "Gaussian basis sets for use in correlated molecular calculations. IV. Calculation of static electrical response properties," J. Chem. Phys. 100, 2975-2988 (1994).

[16] J. R. Hammond, W. A. de Jong, and K. Kowalski, "Coupled-cluster dynamic polarizabilities including triple excitations," J. Chem. Phys. 128, 224102 (2008).

[17] J. R. Hammond, N. Govind, K. Kowalski, J. Autschbach, and S. S. Xantheas, "Accurate dipole polarizabilities for water clusters $n=2-12$ at the coupled-cluster level of theory and benchmarking of various density functionals," 
J. Chem. Phys. 131, 214103 (2009).

[18] K. U. Lao, J. Jia, R. Maitra, and R. A. DiStasio Jr., "On the geometric dependence of the molecular dipole polarizability in water: A benchmark study of higherorder electron correlation, basis set incompleteness error, core electron effects, and zero-point vibrational contributions," J. Chem. Phys. 149, 204303 (2018).

[19] Y. Yang, K. U. Lao, D. M. Wilkins, A. Grisafi, M. Ceriotti, and R. A. DiStasio Jr., "Quantum mechanical static dipole polarizabilities in the QM7b and AlphaML showcase databases," Sci. Data 6, 1-10 (2019).

[20] D. M. Bishop, "Molecular vibrational and rotational motion in static and dynamic electric fields," Rev. Mod. Phys. 62, 343 (1990).

[21] S. Kümmel, J. Akola, and M. Manninen, "Thermal expansion in small metal clusters and its impact on the electric polarizability," Phys. Rev. Lett. 84, 3827 (2000).

[22] U. Hohm, "Experimental static dipole-dipole polarizabilities of molecules," J. Mol. Struct. 1054, 282-292 (2013).

[23] H. B. G. Casimir and B. Polder, "The influence of retardation on the London-van der Waals forces," Phys. Rev. 73, 360-372 (1948).

[24] V. V. Gobre and A. Tkatchenko, "Scaling laws for van der Waals interactions in nanostructured materials," Nat. Commun. 4, 2341 (2013).

[25] A. Ambrosetti, N. Ferri, R. A. DiStasio Jr., and A. Tkatchenko, "Wavelike charge density fluctuations and van der Waals interactions at the nanoscale," Science 351, 1171-1176 (2016).

[26] Y. Yang, K. U. Lao, and R. A. DiStasio Jr., "Influence of pore size on the van der Waals interaction in twodimensional molecules and materials," Phys. Rev. Lett. 122, 026001 (2019).

[27] E. R. Johnson and A. D. Becke, "A post-Hartree-Fock model of intermolecular interactions: Inclusion of higherorder corrections," J. Chem. Phys. 124, 174104 (2006).

[28] J. Tao, J. P. Perdew, and A. Ruzsinszky, "Long-range van der Waals attraction and alkali-metal lattice constants," Phys. Rev. B 81, 233102 (2010).

[29] J. Tao, J. Yang, and A. M. Rappe, "Dynamical screening of van der Waals interactions in nanostructured solids: Sublimation of fullerenes," J. Chem. Phys. 142, 164302 (2015).

[30] T. Gould, K. Simpkins, and J. F. Dobson, "Theoretical and semiempirical correction to the long-range dispersion power law of stretched graphite," Phys. Rev. B 77, 165134 (2008).

[31] L. Spanu, S. Sorella, and G. Galli, "Nature and strength of interlayer binding in graphite," Phys. Rev. Lett. 103, 196401 (2009).

[32] Y. J. Dappe, J. Ortega, and F. Flores, "Intermolecular interaction in density functional theory: Application to carbon nanotubes and fullerenes," Phys. Rev. B 79, 165409 (2009).

[33] S. Lebègue, J. Harl, T. Gould, J. G. Ángyán, G. Kresse, and J. F. Dobson, "Cohesive properties and asymptotics of the dispersion interaction in graphite by the random phase approximation," Phys. Rev. Lett. 105, 196401 (2010).

[34] A. J. Misquitta, J. Spencer, A. J. Stone, and A. Alavi, "Dispersion interactions between semiconducting wires," Phys. Rev. B 82, 075312 (2010).

[35] A. J. Misquitta, R. Maezono, N. D. Drummond, A. J.
Stone, and R. J. Needs, "Anomalous nonadditive dispersion interactions in systems of three one-dimensional wires," Phys. Rev. B 89, 045140 (2014).

[36] P. S. Venkataram, J. Hermann, A. Tkatchenko, and A. W. Rodriguez, "Unifying microscopic and continuum treatments of van der Waals and Casimir interactions," Phys. Rev. Lett. 118, 266802 (2017).

[37] A. Ruzsinszky, J. P. Perdew, J. Tao, G. I. Csonka, and J. M. Pitarke, "van der Waals coefficients for nanostructures: Fullerenes defy conventional wisdom," Phys. Rev. Lett. 109, 233203 (2012).

[38] J. Kauczor, P. Norman, and W. A. Saidi, "Nonadditivity of polarizabilities and van der Waals $C_{6}$ coefficients of fullerenes," J. Chem. Phys. 138, 114107 (2013).

[39] W. A. Saidi and P. Norman, "Polarizabilities and van der Waals $C_{6}$ coefficients of fullerenes from an atomistic electrodynamics model: Anomalous scaling with number of carbon atoms," J. Chem. Phys. 145, 024311 (2016).

[40] J. Tao, Y. Jiao, Y. Mo, Z. H. Yang, J. X. Zhu, P. Hyldgaard, and J. P. Perdew, "First-principles study of the binding energy between nanostructures and its scaling with system size," Phys. Rev. B 97, 155143 (2018).

[41] A. Jiemchooroj, P. Normana, and B. E. Sernelius, "Complex polarization propagator method for calculation of dispersion coefficients of extended $\pi$-conjugated systems: The $C_{6}$ coefficients of polyacenes and $\mathrm{C}_{60}$," J. Chem. Phys. 123, 124312 (2005).

[42] K. Kowalski, J. R. Hammond, W. A. de Jong, and A. J. Sadlej, "Coupled cluster calculations for static and dynamic polarizabilities of $\mathrm{C}_{60}$," J. Chem. Phys. 129, 226101 (2008).

[43] D. H. Friese, N. O. C. Winter, P. Balzerowski, R. Schwan, and C. Hättig, "Large scale polarizability calculations using the approximate coupled cluster model CC2 and MP2 combined with the resolution-of-the-identity approximation," J. Chem. Phys. 136, 174106 (2012).

[44] R. Antoine, Ph. Dugourd, D. Rayane, E. Benichou, and M. Broyer, "Direct measurement of the electric polarizability of isolated $\mathrm{C}_{60}$ molecules," J. Chem. Phys. 110, 9771-9772 (1999).

[45] A. Ballard, K. Bonin, and J. Louderback, "Absolute measurement of the optical polarizability of $\mathrm{C}_{60}$," J. Chem. Phys. 113, 5732-5735 (2000).

[46] I. Compagnon, R. Antoine, M. Broyer, Ph. Dugourd, J. Lermé, and D. Rayane, "Electric polarizability of isolated C70 molecules," Phys. Rev. A 64, 025201 (2001).

[47] M. Berninger, A. Stefanov, S. Deachapunya, and M. Arndt, "Polarizability measurements of a molecule via a near-field matter-wave interferometer," Phys. Rev. A 76, 013607 (2007).

[48] A. Kumar and A. J. Thakkar, "Dipole polarizability, sum rules, mean excitation energies, and long-range dispersion coefficients for buckminsterfullerene $\mathrm{C}_{60}$," Chem. Phys. Lett. 516, 208-211 (2011).

[49] Y. Y. Fein, P. Geyer, F. Kiałka, S. Gerlich, and M. Arndt, "Improved accuracy fullerene polarizability measurements in a long-baseline matter-wave interferometer," Phys. Rev. Res. 1, 033158 (2019).

[50] G. K. Gueorguiev, J. M. Pacheco, and D. Tománek, "Quantum size effects in the polarizability of carbon fullerenes," Phys. Rev. Lett. 92, 215501 (2004).

[51] A. A. Lucas, L. Henrard, and Ph. Lambin, "Computation of the ultraviolet absorption and electron inelastic 
scattering cross section of multishell fullerenes," Phys. Rev. B 49, 2888 (1994).

[52] J. Tao, J. P. Perdew, and A. Ruzsinszky, "Accurate van der Waals coefficients from density functional theory," Proc. Natl. Acad. Sci. USA 109, 18-21 (2012).

[53] J. P. Perdew, J. Tao, P. Hao, A. Ruzsinszky, G. I. Csonka, and J. M. Pitarke, "Spherical-shell model for the van der Waals coefficients between fullerenes and/or nearly spherical nanoclusters," J. Phys. Condens. Matter 24, 424207 (2012).

[54] J. Tao and J. P. Perdew, "Communication: Nonadditivity of van der Waals interactions between nanostructures," J. Chem. Phys. 141, 141101 (2014).

[55] J. Tao, Y. Fang, P. Hao, G. E. Scuseria, A. Ruzsinszky, and J. P. Perdew, "van der Waals coefficients beyond the classical shell model," J. Chem. Phys. 142, 024312 (2015).

[56] J. Tao, Y. Mo, G. Tian, and A. Ruzsinszky, "Accurate van der Waals coefficients between fullerenes and fullerene-alkali atoms and clusters: Modified singlefrequency approximation," Phys. Rev. B 94, 085126 (2016).

[57] J. Tao and A. M. Rappe, "Accurate higher-order van der Waals coefficients between molecules from a model dynamic multipole polarizability," J. Chem. Phys. 144, 031102 (2016).

[58] P. W. Fowler, "Vibration-rotation effects on properties of symmetric tops and linear molecules," Mol. Phys. 43, 591-600 (1981).

[59] P. W. Fowler, "Vibrational effects on properties of spherical tops," Mol. Phys. 51, 1423-1428 (1984).

[60] A. J. Russell and M. A. Spackman, "Vibrational averaging of electrical properties: Development of a routine theoretical method for polyatomic molecules," Mol. Phys. 84, 1239-1255 (1995).

[61] K. Hui and J.-D. Chai, "SCAN-based hybrid and doublehybrid density functionals from models without fitted parameters," J. Chem. Phys. 144, 044114 (2016).

[62] J. Sun, A. Ruzsinszky, and J. P. Perdew, "Strongly constrained and appropriately normed semilocal density functional," Phys. Rev. Lett. 115, 036402 (2015).

[63] H. Prinzbach, A. Weiler, P. Landenberger, F. Wahl, J. Wörth, L. T. Scott, M. Gelmont, D. Olevano, and B. von Issendorff, "Gas-phase production and photoelectron spectroscopy of the smallest fullerene, $\mathrm{C}_{20}$," Nature 407, 60 (2000).

[64] Y. Shao et al., "Advances in molecular quantum chemistry contained in the Q-Chem 4 program package," Mol. Phys. 113, 184-215 (2015).

[65] V. Blum, R. Gehrke, F. Hanke, P. Havu, V. Havu,
X. Ren, K. Reuter, and M. Scheffler, "Ab initio molecular simulations with numeric atom-centered orbitals," Comput. Phys. Commun. 180, 2175-2196 (2009).

[66] J. P. Perdew, A. Ruzsinszky, J. Sun, S. Glindmeyer, and G. I. Csonka, "van der Waals interaction as a summable asymptotic series," Phys. Rev. A 86, 062714 (2012).

[67] The outer physical radius was estimated via $\langle R\rangle=\langle r\rangle+$ $t / 2$, in which $\langle r\rangle$ is the average distance from each atom to the nuclear center of charge and $t=3.4$ Bohr [50, 51].

[68] G. F. Thomas, F. Mulder, and W. J. Meath, "Isotropic $C_{6}, C_{8}$ and $C_{10}$ interaction coefficients for $\mathrm{CH}_{4}, \mathrm{C}_{2} \mathrm{H}_{6}$, $\mathrm{C}_{3} \mathrm{H}_{8}, \mathrm{n}-\mathrm{C}_{4} \mathrm{H}_{10}$ and cyclo- $\mathrm{C}_{3} \mathrm{H}_{6}$," Chem. Phys. 54, 45-54 (1980).

[69] D. M. Wilkins, A. Grisafi, Y. Yang, K. U. Lao, R. A. DiStasio Jr., and M. Ceriotti, "Accurate molecular polarizabilities with coupled cluster theory and machine learning," Proc. Natl. Acad. Sci. USA 116, 3401-3406 (2019).

[70] For $C_{n}$, the choice of $R=\langle R\rangle$ overestimates $C_{6}$ and $C_{8}$ by $14.2 \%$ and $5.7 \%$, and underestimates $C_{10}$ by $2.4 \%$.

[71] P. Lambin, A. A. Lucas, and J.-P. Vigneron, "Polarization waves and van der Waals cohesion of $\mathrm{C}_{60}$ fullerite," Phys. Rev. B 46, 1794 (1992).

[72] D. R. Penn, "Wave-number-dependent dielectric function of semiconductors," Phys. Rev. 128, 2093 (1962).

[73] M. Rahm, R. Hoffmann, and N. W. Ashcroft, "Atomic and ionic radii of elements 1-96," Chem. Eur. J. 22, 14625-14632 (2016).

[74] D. V. Fedorov, M. Sadhukhan, M. Stöhr, and A. Tkatchenko, "Quantum-mechanical relation between atomic dipole polarizability and the van der Waals radius," Phys. Rev. Lett. 121, 183401 (2018).

[75] L. T. Chadderton, "Axisymmetric vibrational modes of fullerene $\mathrm{C}_{60}$, , J. Phys. Chem. Solids 54, 1027-1033 (1993).

[76] H. Eisler, S. Gilb, F. H. Hennrich, and M. M. Kappes, "Low frequency raman active vibrations in fullerenes. 1. Monopolar modes," J. Phys. Chem. A 104, 1762-1768 (2000).

[77] H.-J. Eisler, F. H. Hennrich, S. Gilb, and M. M. Kappes, "Low frequency raman active vibrations in fullerenes. 2. Quadrupolar modes," J. Phys. Chem. A 104, 1769-1773 (2000).

[78] D. Jing and Z. Pan, "Molecular vibrational modes of $\mathrm{C}_{60}$ and $\mathrm{C}_{70}$ via finite element method," Eur. J. Mech. ASolid 28, 948-954 (2009).

[79] K. A. Makhnovets and A. K. Kolezhuk, "Finite-size nanowire at a surface: Unconventional power laws of the van der Waals interaction," Phys. Rev. B 96, 125427 (2017). 